\title{
Unlocking the power of population health cohort studies with biobanking in Australia
}

\section{Ashleigh A Armanasco ${ }^{a, d}$, Alvin Lee ${ }^{b}$, Bette Liuc, Leigh Mclndoe ${ }^{b}$ and Martin McNamarab}

a NSW Public Health Training Program, Centre for Epidemiology and Evidence, NSW Ministry of Health, Sydney, Australia

b Sax Institute, Sydney, NSW, Australia

c School of Public Health and Community Medicine, Faculty of Medicine, UNSW Sydney, Australia

${ }^{d}$ Corresponding author: Ashleigh.Armanasco@health.nsw.gov.au

\section{Article history}

Publication date: 13 December 2022

Citation: Armanasco AA, Lee A, Liu B, Mclndoe L, McNamara M. Unlocking the power of population health cohort studies with biobanking in Australia. Public Health Res Pract. 2022; 32(4):e32012201. First published 10 February 2022. https://doi. org/10.17061/phrp32012201

\section{Key points}

- Population-scale biobanks enhance opportunities to study disease and population health by enabling access to biomedical information that is linked with health, lifestyle and environmental data

- Nesting biospecimen collection in cohort studies enables us to address research questions that were previously unanswerable

- Although there are practical and ethical challenges related to population biobanking research, Australian cohort studies such as the 45 and Up Study can be enriched by biospecimen collection, because of their size, quality of data collected, and existing governance mechanisms

\section{Abstract}

Biobanks have great potential in advancing population health research by enabling access to biospecimens that can be linked to health data. Governments and research institutes worldwide have made large commitments to biobanking in recent years, often through combining cohort studies and biospecimen collections. We aim to explore the opportunities and challenges of establishing a population-scale biobank in Australia. Using existing longitudinal cohort studies to collect biospecimens can be an efficient way to rapidly advance research infrastructure in Australia. The 45 and Up Study is a large-scale longitudinal cohort with self-reported health, behaviour and lifestyle data, and may be one suitable candidate to include biospecimen collections to contribute to a population biobank. However, there are a number of ethical and practical issues specific to biospecimen collections, such as ensuring the privacy and rights of participants are protected, and ensuring sufficient resourcing to support infrastructure. Overcoming these challenges and bringing together diverse fields of expertise will enable us to maximise the potential of cohort studies and biobanks. In combination, these data sources can efficiently advance population health research beyond what is achievable with either of these resources separately.

\section{Background}

Biobanks are collections of biospecimens that have been gathered for research purposes. They vary in scope, ranging from small collections derived from clinically selected samples through to prospective population cohorts. ${ }^{1,2}$ Linking health administrative data with survey data is a wellestablished method to study emerging health trends and problems in a cohesive and comprehensive way, however linking data derived from biospecimen samples with these datasets is often considered the next frontier in population health. ${ }^{3,4}$

Integrating biomedical data, including genomic information, with population health data will provide opportunities to gain a more complete understanding of factors that influence health outcomes and address a 
much wider range of questions than either data source can offer independently. ${ }^{1}$ For example, we can explore the role of genetics in the relationship between physical activity, nutrition and obesity by linking and analysing genomic, self-reported, Medicare and hospitalisation data. Or we can research the impact of the proteome on the effectiveness of pharmacotherapy for different diseases and disorders by analysing proteomic and linked Australian Pharmaceutical Benefits Scheme data (on subsidised medication use) to inform an innovative and personalised approach to disease treatment. ${ }^{1}$ There is immense potential in coupling genomic information with health and lifestyle data to advance our knowledge of health and disease to ultimately influence health outcomes. ${ }^{6,7}$ However, we need large numbers of participants to represent diverse populations and sufficiently power such research. ${ }^{2}$

Globally, there is high-level support to establish population biobanks. The Organisation for Economic Co-operation and Development was one of the first organisations to advocate the importance of biobanks in 2006. ${ }^{8}$ In the following years, various countries began establishing biobanks as major research infrastructure. The UK Biobank and the US Precision Medicine Initiative are just two examples of national commitments to biobanking infrastructure. ${ }^{6,9}$ Collectively, these resources will include biospecimens (primarily blood samples) from 1.5 million people together with self-reported health and lifestyle information, and linked administrative data.

Currently, the majority of established Australian biobanks use clinically derived samples from participants with a particular illness, such as cancer. ${ }^{10}$ Although these are useful resources, for population health to fully leverage the potential of biobanking, we need samples that inform us about the broader population. The 2016 national research infrastructure roadmap identified Australia would benefit from a population biobank, similar to other population biobanks in the US and UK. ${ }^{11}$ The establishment of the NSW Health Statewide Biobank in 2017 was an important step towards achieving this vision. ${ }^{12}$ This biobank provides facilities for storage and processing of biospecimens, with the capacity to hold millions of samples. ${ }^{12}$ There has also been substantial investment in Australia in genomics translation and research using linked data. In 2017-18, the Australian Government committed $\$ 500$ million over 10 years to the Australian Genomics Health Futures Mission and $\$ 80$ million over 8 years to enable the development of data registries, biobanks and data linkage platforms to support Australian medical research. ${ }^{13}$

\section{The potential for cohort studies in biobanking}

Biobanking can be used to investigate the interactions between biology, genetics, environment, behaviour and health outcomes. Prospective cohort study designs have several benefits for biobanking research. Cohort studies typically include large numbers of participants who have agreed to be followed up over time to allow the study of multiple outcomes. The prospective design also enables research on disease predictors, because biospecimens can be collected before the onset of illness. Other study designs such as case-control studies are often used to examine interactions between genetic information and other factors such as environmental and lifestyle factors, however these can introduce significant sources of bias due to control selection. ${ }^{14}$ Use of prospective designs can reduce the likelihood of biases such as subject selection biases and recall bias.

Internationally, researchers are taking advantage of the benefits of integrating prospective designs and biospecimen collection. There are a growing number of large prospective longitudinal cohort studies that include biospecimens, for example in Norway ${ }^{15}$, the $\mathrm{UK}^{2,16}$ and Taiwan. ${ }^{17}$ These collections support the integration of 'omics' (e.g. genomics, proteomics and metabolomics) and biomarker data with epidemiological research.

\section{Challenges for population biobanking in Australia}

There are a number of practical and ethical challenges related to population biobanking research. There are significant costs associated with the establishment and maintenance of both large-scale cohort studies and biospecimen collections. Leveraging existing cohort studies in Australia would be one way to efficiently meet the need for a population biobank ${ }^{11}$ while also gaining the benefits of a large-scale prospective design. Using an existing cohort study reduces some of the establishment costs as there is an existing pool of participants, data management systems and governance structures in place. Despite this, we still need to consider the most appropriate forms of funding to ensure a population biospecimen collection is sustainable. The majority of international biobanks have received some form of government funding or support from charitable, academic or research organisations. Many biobanks also have a cost-recovery model, but it has been noted these models alone are insufficient to cover running costs. ${ }^{18}$

Biobanks are increasingly facing pressure to commercialise and translate outcomes, thus increasing expectations of industry partnerships. These partnerships are often necessary and can foster translation of useful technologies and practices, however commercialisation of biobanks raises conflict of interest issues. These include: the potential to adversely impact public trust; consent challenges (such as the possible requirement to obtain re-consent); privacy issues; and misaligned objectives between public and private partners. Effective governance and cross-sector collaboration, including with government, will be part of the solution to these challenges. 
We need to build datasets that are sufficiently interoperable with other biobanks to allow data to be linked. We also need to consider differences in privacy laws across international jurisdictions to maintain participants' rights. There are several organisations and networks that are working to overcome these challenges and facilitate sharing data between researchers and biobanks. Globally there are several examples of organisations that focus on fostering networks and harmonising biobanking approaches such as the International Society for Biological and Environmental Repositories and the International HundredK+ Cohorts Consortium. ${ }^{19,20}$ The Australasian Biospecimen Network Association supports and promotes best-practice biobanking in Australasia. ${ }^{21}$ In NSW, clinicians and researchers are working together through the Sydney Genomics Collaborative ${ }^{22}$ and there are already established policies to govern population biospecimen collections, access to biobanks and data linkage. .3,24 $^{23}$ Guidelines to ensure protection of participants' interests and privacy have also been considered nationally and globally. 19,25 National and international networks will continue to guide governance of population biobanks in Australia and provide advice and mechanisms to protect the privacy of participants when sharing and combining data across international jurisdictions. ${ }^{26}$

\section{The potential of biobanking in}

\section{Australia}

Longitudinal cohort studies should consider the benefits of collecting biospecimens from participants to enable future research. Incorporating biospecimens into existing large-scale cohort studies would extend their utility to new fields, such as the proliferating 'omics' fields and would capitalise on the potential to support biobanking within Australia.

The Sax Institute's 45 and Up Study ("Study") previously investigated the characteristics of Australian cohort study participants who do and do not take up an additional invitation to join a long-term biobank. ${ }^{27}$ The research found that the uptake of the invitation to those who were already enrolled in the Study was approximately $33 \%{ }^{27}$, with collected samples being used to develop a Medical Genome Reference Bank. ${ }^{28}$ The Study is a longitudinal cohort study of people aged 45 years and over (at baseline) residing in NSW, Australia. Prospective participants were randomly sampled from the Department of Human Services (formerly Medicare Australia) enrolment database, which provides near complete coverage of the population. A total of 267153 participants joined the Study at the time of recruitment in 2006-2009 by completing a baseline questionnaire and giving signed consent for follow-up and linkage of their information to routine health databases. About $18 \%$ of those invited participated and participants included about $11 \%$ of the NSW population in this age group. ${ }^{29}$
The Study's large participant base, extensive existing data on participants and established processes for data sharing and linkage make it a highly suitable candidate to contribute to an Australian population biobank. The Sax Institute is currently piloting the most practical ways to collect blood samples from consenting participants using NSW Health public pathology services. Findings from the pilot, including barriers and potential unintended consequences, are expected to be published in the near future.

While the Study remains the largest longitudinal cohort study in Australia and has been established as an open-access resource, it focuses on an ageing cohort as participants in the study are now aged at least 55 years and mainly residing in NSW. ${ }^{29}$ Other large Australian longitudinal cohort studies will need to also consider how they can contribute to population biobanking if we are to develop a truly representative national biobank.

\section{Conclusions}

Biospecimen collections and prospective cohort studies can have a mutually beneficial relationship in biobanking, both enabling the other to enhance our understanding of the complex interactions underpinning health outcomes. Australia has developed much of the infrastructure needed to support a population biobank. As Australia's largest longitudinal cohort study, the 45 and Up Study is in a strong position to be enriched by the addition of biospecimens to bridge the population biobank gap in Australian research infrastructure. However, other longitudinal cohort studies will also need to contribute if we are to ensure diversity of a national collection. Even still, we will need to carefully consider how we approach the inclusion of biospecimens to support collaboration and address ethical issues. Overcoming these challenges is a worthwhile endeavour; the addition of biobanking to population cohort studies has the potential to unlock a wide range of answers and push the boundaries of our current knowledge.

\section{Acknowledgements}

This work was completed while author AA was employed as a trainee on the Public Health Training Program funded by the NSW Ministry of Health. She undertook this work while based at the Sax Institute.

This paper is part of a special issue of PHRP reflecting on the 45 and Up Study. The edition is published in memory of world-renowned epidemiologist Dame Valerie Beral (1946-2022), who played a vital role in establishing and supporting the Study.

The 45 and Up Study is managed by the Sax Institute in collaboration with partners Cancer Council NSW, the Heart Foundation and NSW Ministry of Health. We thank the many thousands of people participating in the 45 
and Up Study. Author MM is the Chief Investigator of the Study.

The authors thank Professor Bruce Armstrong for his expert advice and Emilie Kirkeby-Garstad for assistance with editing and referencing.

The conduct of the 45 and Up Study has been approved by the UNSW Sydney Human Research Ethics Committee (HREC).

\section{Peer review and provenance}

Externally peer reviewed, not commissioned.

\section{Competing interests}

None declared.

\section{Author contributions}

AA was responsible for the drafting and editing the manuscript. AL was responsible for review and editing the manuscript. BL was responsible for providing expert advice, reviewing and editing the manuscript. LM was responsible for drafting sections, reviewing and editing the manuscript. MM was responsible for the concept and review of the manuscript.

\section{References}

1. Coppola L, Cianflone A, Grimaldi AM, Incoronato M, Bevilacqua $P$, Messina $F$, et al. Biobanking in health care: evolution and future directions. J Transl Med. 2019;17(1):1-8.

2. Harris JR, Burton P, Knoppers BM, Lindpaintner K, Bledsloe M, Brookes AJ, et al. Toward a roadmap in global biobanking for health. Eur J Hum Genet. 2012;20(11):1105-11.

3. Bureau of Health Information. Data matters. Linking data to unlock information. The use of linked data in healthcare performance assessment. Sydney; BHI; 2015. [cited 2021 Sep 23]. Available from: www.bhi.nsw.gov.au/_ data/assets/pdf_file/0003/290712/DataMatters.pdf

4. Olver I. Linking data to improve health outcomes. Med J Aust. 2014;200(7):368-9.

5. Zielhuis GA. Biobanking for epidemiology. Public Health. 2012;126(3):214-6.

6. Palmer LJ. UK Biobank: bank on it. Lancet. 2007;369(9578):1980-2.

7. Collins FS, Green ED, Guttmacher AE, Guyer MS. A vision for the future of genomics research. Nature. 2003;422(6934):835-47.
8. Organisation for Economic Co-operation and Development (OECD). Creation and governance of human genetic research databases. Paris: OECD Publishing; 2006 [cited 2022 Jan 11]. Available from: www.oecd.org/sti/emerging-tech/creation-andgovernance-of-human-genetic-research-databases.htm

9. Collins FS, Varmus $\mathrm{H}$. A new initiative on precision medicine. N Engl J Med. 2015;372(9):793-5.

10. Victorian Cancer Biobank, Victorian Cancer Biobank. Melbourne, Victoria: VBC; 2018 [cited 2020 Jan 26]. Available from: viccancerbiobank.org.au/

11. Australian Government. 2016 national research infrastructure roadmap. Canberra: Australian Government; 2017. p.98 [cited 2022 Jan 11]. Available from: www.chiefscientist.gov.au/sites/default/files/ED160269-National-Research-Infrastructure-Roadmap-Report_ Internals_ACC.pdf

12. NSW Health. $\$ 12$ million Biobank to revolutionise research. Sydney NSW: NSW Health; 2017 [cited 2019 Mar 28]. Available from: www.health.nsw.gov.au/news/ Pages/20171113_00.aspx

13. Australian Government Department of Health. Medical Research Future Fund (MRFF) 10-year investment plan. Canberra: Australian Government; 2019 [cited 2021 Sep 23]. Available from: www.health.gov.au/resources/ publications/medical-research-future-fund-mrff-10-yearinvestment-plan

14. Manolio T, Bailey-Wilson J, Collins F. Genes, environment and the value of prospective cohort studies. Nat Rev Genet. 2006;7(10):812-20.

15. Magnus $P$, Holmen J. Modern biobanks in Norway. Nor Epidemiol. 2015;25(1-2):47-52.

16. Fraser A, Macdonald-Wallis C, Tilling K, Boyd A, Golding J, Davey Smith G, et al. Cohort profile: the Avon Longitudinal Study of Parents and Children: ALSPAC mothers cohort. Int J Epidemiol. 2012;42(1):97-110.

17. Fan CT, Lin JC, Lee CH. Taiwan Biobank: a project aiming to aid Taiwan's transition into a biomedical island Pharmacogenomics. 2008;9(2):265-46.

18. Chalmers D, Nicol D, Kaye J, Bell J, Campbell AV, Ho CW, et al. Has the biobank bubble burst? Withstanding the challenges for sustainable biobanking in the digital era. BMC Med Ethics. 2016;17(1):39.

19. International Society for Biological and Environmental Repositories (ISBER). About ISBER. Vancouver, Canada: ISBER; 2018 [cited 2019 Oct 21]. Available from: www. isber.org/page/About

20. International HundredK+ Cohorts Consortium (IHCC). About IHCC. North Carolina, US: IHCC; 2020 [cited 2020 Sep 21]. Available from: www.inccglobal.org/about/

21. Australasian Biospecimen Network Association (ABNA). Who are ABNA? Melbourne, Australia: ABNA; 2019 [cited 2019 Oct 21]. Available from: abna.org.au/ 
22. Sydney Genomics Collaborative. About. Sydney, Australia: Sydney Genomics Collaborative; 2019 [cited 2019 Oct 21]. Available from: sgc.garvan.org.au/about

23. Statewide Biobank. Access policy for NSW Health strategic collections. Sydney: NSW Health; 2020 [cited 2020 Sep 17]. Available from: www.biobank.health.nsw. gov.au/wp-content/uploads/2020/06/NSWHSB-290620Strategic-Collection-Access-Policy-FINAL_.pdf

24. Health Statewide Biobank. NSW Health consent toolkit. Sydney: NSW Health; 2020 [cited 2020 Sep 17].

Available from: biobank.health.nsw.gov.au/wp-content/ uploads/2020/07/NSW-Health-Consent-Toolkit-V3-072020. pdf

25. National Health and Medical Research Council (NHMRC). Biobanks information paper. Canberra: NHMRC; 2010 [cited 2020 Sep 17]. Available from: www.nhmrc.gov.au/ about-us/publications/biobanks-information-paper
26. Chalmers D. Biobanking and privacy laws in Australia. J Law Med Ethics. 2015;43(4):703-13.

27. Banks E, Herbert N, Mather T, Rogers K, Jorm L. Characteristics of Australian cohort study participants who do and do not take up an additional invitation to join a long-term biobank: the 45 and Up Study. BMC Res Notes. 2012;5(1):655.

28. Pinese M, Lacaze P, Rath EM, Stone A, Brion MJ; Aeur A, et al. The Medical Genome Reference Bank contains whole genome and phenotype data of 2570 healthy elderly. Nat Commun. 2020;11(1):435.

29. 45 and Up Study Collaborators. Cohort profile: the 45 and Up Study. Int J Epidemiol. 2007;37(5):941-7.

\section{Copyright: (c)(1)}

(C) 2022 Armanasco et al. This article is licensed under the Creative Commons Attribution-NonCommercial-ShareAlike 4.0 International Licence, which allows others to redistribute, adapt and share this work non-commercially provided they attribute the work and any adapted version of it is distributed under the same Creative Commons licence terms. See: www.creativecommons.org/licenses/by-nc-sa/4.0/ 